\title{
Revisión Siatemática: hydergina para el tratamiento de la demencia
}

Hydergine for dementia (Cochrane Review). Olin J, Schneider L, Novit A, Luczak S. Cochrane Library, Issue 3, 2001. Oxford: Update Software; 357:89-95

\section{Objetivo}

Evaluar la eficacia reportada de la Hydergina en pacientes con posible demencia e investigar los potenciales moderadores de este efecto.

\section{Diseño y estrategia de búsqueda}

Metanálisis de trabajos doble ciegos, aleatorizados con grupo control y comparados con placebo para el tratamiento con hydergina por más de una semana en sujetos con demencia o síntomas compatibles con demencia.

Todos fueron identificados a través de las palabras "hydergin", "ergoloid" y "dihydroergo" usando 17 bases de datos en idioma inglés.

\section{Selección de los estudios}

A través del título y el resumen, un revisor descartó las citas irrelevantes y posteriormente otros dos, trabajando cada uno por separado, seleccionaron los trabajos a analizar. Los conflictos fueron resueltos por el primero.

Los mismos revisores evaluaron la calidad metodológica de cada trabajo usando las guías de la Colaboración Cochrane, y se establecieron categorías en función de la asignación e implementación del tratamiento con hydergina: adecuada, intermedio, inadecuada.

\section{Recolección y análisis de los Datos}

Diecinueve trabajos reunieron los criterios de inclusión. El numero de participantes osciló entre 19 hasta 458. Dieciocho estudios compararon hydergina contra placebo y uno, placebo con polivitaminas.
Las dosis de hydergina también varió entre los diferentes trabajos entre de $1.5 \mathrm{mg} / \mathrm{día}$ hasta $7.5 \mathrm{mg} /$ día. Los resultados fueron medidos por la mejoría clínica en Escalas de Puntuación Integral (EPI) y Escalas de Impresión Clínica Global (ICG). Como sólo siete trabajos emplearon pruebas neuropsicológicas, estos resultados no pudieron ser utilizado para el análisis. Otras variables analizadas fueron la duración de los trabajos, la edad, el sexo, las dosis utilizadas, si el paciente estaba internado o era atendido en forma ambulatoria, etc.

\section{Resultados Principales}

De los 19 trabajos, 13 proveían información en escalas de ICG, 9 en EPI y 3 en ambas escalas.

Para los que utilizaron las escalas globales hubo un efecto significativo a favor de la Hydergyna con un OR de 3,78 (IC95\%: 2,72$5,27)$.

Con respecto a las demás variables no se encontró diferencias estadísticamente significativas pero si una tendencia a poseer mayor efectos las dosis altas en pacientes mas jóvenes (65 a 75 vs mayores de 75)

La hydergina fue en general bien tolerada y los efectos adversos sólo aparecieron con altas dosis y en pacientes jóvenes.

\section{Conclusiones}

El uso de hydergina muestra un efecto significativo en el tratamiento de los problemas cognitivos cuando son evaluados por escalas globales. El pequeño número de trabajos aceptables para el análisis limitan esta conclusión.

\section{Comentario}

Los problemas de la memoria en los ancianos son frecuentes en la atención ambulatoria llegando al $30 \%$ de todas las consultas en algunas series (1). Una declinación de la memoria y de la velocidad de procesamiento de la información ocurre normalmente con la edad pero no afecta la vida diaria y en general no progresa. En cambio, la demencia y en especial la Enfermedad de Alzheimer (EA), representan un daño cognitivo persistente y progresivo que afecta a más de 15 millones de personas en todo el mundo. En este contexto, la demanda de "pastillas para la memoria" aparece no pocas veces en un consultorio de atención primaria (2).

La Hydergina es una combinación de cuatro derivados de la ergotoxina que se usa desde 1949 en el tratamiento de la demencia, de los olvidos asociados a la edad y de los acúfenos. Si bien su uso para estos fines ha sido muy difundido en las últimas décadas, los médicos generalistas suelen manejar poca información acerca de esta droga.

El metanálisis que hoy comentamos sólo pudo evaluar 19 ensayos clínicos realizados con Hydergina desde 1955 hasta 1990, previamente a que fueran establecidas las definiciones consensuadas de demencias. En consecuencia, son heterogéneos en cuanto a la definición de demencia, los parámetros cognitivos evaluados y la dosis usada de Hydergina, lo que agrega mayor dificultad al análisis.

Los ensayos duraron entre 9 semanas y 15 meses. Los diagnósticos usados para la inclusión también variaron, algunos estudios hablan de Alzheimer o de "otra demencia vascular" y otros usan términos en desuso como "deterioro o insuficiencia cerebral" y "demencia primaria".

Lamentablemente no se pudieron evaluar mejoras en tests neurocognitivos puesto que sólo siete trabajos los utilizaron, no habiendo empleando ninguno el ADAS-cogs (validado y recomendado por la FDA para evaluar nuevos tratamientos en el Alzheimer y otros deterioros cognitivos) $)^{(3)}$.

Como comentan los revisores en sus resultados se observó un efecto favorable del tratamiento con Hydergina en los parámetros evaluados. El beneficio podría ser mayor con dosis superiores a $4.5 \mathrm{mg} / \mathrm{dí}$. No obstante los efectos son modestos, aún con dosis altas, alcanzando significación estadística en sólo la mitad de los estudios analizados (muchos demasiado pequeños para demostrar un eventual efecto)

Conclusión del comentario: Si bien el uso de Hydergina se asoció a algunas mejoras en la puntuación de escalas globales, la interpretación de los resultados debe ser muy cautelosa debido a que no se utilizó una definición común para demencia ni el test reconocido internacionalmente para evaluar los tratamientos farmacológicos de esta entidad. En definitiva, a pesar de los 50 años de experiencia en su uso, no existe evidencia de buena calidad que permita evaluar la eficacia de la Hydergina en el tratamiento de la demencia.

\section{Dr. Manuel Montero-Odasso [ Servicio de Clínica Médica y Sección Geriatría. Hospital Italiano de Buenos Aires ]}

\section{Referencias}

1. Herbert LE, Scherr PA, Beckett LA, et al. Age -specific incidence ogf Alzheimer's disease in a comunity population. JAMA;1995; 273: 1354-1359

2. Mayeux, R and Sano, M. Treatment of Alzheimer's Disease. N EngJ Med 1999; 34: 1670-1679.

3. Leber P. Draft guidelines for the clinical evaluation of antidementia drugs. Washington DC :Food and Drugs Administration, 1990 (FDA publicatin no. F91-19331 